unnecessary publication and also that those possessing special knowledge should use their opportunities to produce works of wide interest, such as guides to the literature and bibliographies.

Mr. K. C. D. Vornon's admirable factual account of the libraries and work of some learned societies began, like Mr. Ardern's paper, with a historical review. Broadly speaking, he suggested, the strength of many of these libraries lies in their collection of periodicals, and he regretted that the holdings of many of these libraries are not recorded in publications such as the "World List of Scientific Periodicals". Some of these libraries have almost unknown riches on their bookshelves, and many have important collections of manuscripts. Many of them, however, are undeveloped and largely unknown except to small groups of people. Their resources could add much more to scholarship ; but although some, particularly those serving expanding professions, have been forging ahead, recataloguing and rearranging their books, binding old books and introducing more upto-date methods, much remain 3 to be done before these libraries are fully mobilized to serve their societies.

\section{THE MELLON INSTITUTE}

\section{REPORT FOR 1955-56}

T HE annual report of the president, Dr. E. R. Weidlein, to the Board of Trustees of the Mellon Institute, Pittsburgh, for the year ended February 29, $1956^{*}$, records that expenditure on pure and applied research was $5,009,213$ dollars, of which $1,125,213$ dollars was in support of original investigations in pure science in the Institute's fiveresearch departments and conducted under the auspices of thirteen fellowships. The report traces the idea of setting up a partnership between American industry and science, which the Institute represents, to Dr. R. K. Duncan who, after attending the Sixth International Congress of Applied Chemistry, held in Rome in 1906, arranged for the first industrial fellowship at the University of Kansas on January 1, 1907. During the past year 160 members of the Institute were engaged in pure science research projects and 412 members on the applied science researches conducted under fifty-eight fellowships. Of these seventy-one fellowships, 44 were multiple and 27 individual; four had been proceeding for forty years, four more for thirty-five years and six for twenty-five years.

Investigations in the Department of Research in Chemical Physics have included a detailed study of sodium, potassium and calcium salts to determine the magnitude of the anion effects in flame photometry, the redetermination of the infra-red spectrum of heavy benzene in both gas and liquid phases, and studies of the effect of temperature on the infra-red spectra of cyclohexane and dioxan; special equipment was assembled for measuring the intensity of X-ray scattering at very small angles. In the Department of Research in Physical Chemistry the study was completed of the effects of packing density and annular width on the separating efficiency of packed thermal diffusion columns, and work was considerably extended in chromatography. Three

* Scientific Research Progress in Mellon Institute, 1955-1956. * Scientifte Research Progress in Mellon Institute, 1955-1956.
(Annual Report Series, No. 43.) Pp. vi +54. (Pittsburgh, Pa. : Mellon (Annual Report high-efficiency distilling units capable of handling charges up to 20 l. have been designed and installed, and a start made on the design of an automatic take-off system. Work on the determination and correlation of physico-chemical properties of hydrocarbons of high molecular weight continued, and the changes in pore-volume distribution of reactivated filtration clays are being followed in co-operation with the Multiple Petroleum Fellowship, and the absorption and desorption phenomena of porous glass in co-operation with the Glass Science Multiple Fellowship.

There is a continuing demand for workers in the Department of Instrumentation to construct instruments previously developed in it or by others, and also for training in the operation of instruments. In a basic study of the recording micro-balance and weighing techniques a procedure has been developed to adjust the instrument to be very insensitive to the tilt of the building and balance table. In the Department of Applied Mathematics a study has been made of the mathematical models used in predicting the concentration of atmospheric pollution; work has also been begun in the Department on programming and coding a routine for calculating structure factors used in crystal-structure analysis by the Department of Research in Chemical Physics, and assistance has been given the Multiple Glass Science Fellowship in determining light scattering in glass as a function of fluctuations in anisotropy and density and to the Multiple Petroleum Fellowship on statistical analysis and experimental design in the engine testing of petrol and oils.

Following preliminary studies, in co-operation with the Multiple Fellowship on Petroleum, with a Lingane. type of potentiostat, the Department of Analytical Chemistry has provided a more widely adaptable instrument for coulometric analyses at controlled potentials and has expanded its microbiological and microscopical services during the year. A new microtome permits the preparation of cross-sections of biological and inanimate materials as thin as $0.05 \mu$, and a new stereoscopic microscope is an invaluable aid in selecting areas for photomicrography and for the examination of surfaces. A study of the effeet of micro-organisms on petroleum and petroleum products was commenced, and procedures were formulated for cultivating and counting microorganisms in aerosol dispersions. The expansion characteristics of starches and flours were studied in co-operation with the Food Varieties Multiple Fellowship, and the fundamental study of the chemo-synthetic autotrophic bacterium, which has been designated Ferrobacillus ferroxidans, isolated from bituminous coal-mines was continued.

Reviewing the work carried out under the Fellowships in Pure Science, the report stresses the work of the Industrial Hygiene Foundation, which has now completed twenty years of service to industry, including 380 companies in its membership, for which seven hundred special studies have been made as well as fifty basic research programmes in the Foundation's laboratories. Field investigations during the year included the determination of the magnitude of the in-plant contamination of the air with hexavalent chromium compounds, and the magnitude of fluoride exposures in specific areas of a chemical plant. A study was being made of the incidence of occupational dermatitis due to specific substances and the importance of these substances as causative agents in industrial dermatitis, and another 
of the effects of abrasive dust on normal lungs and on the evolution of pulmonary tuberculosis. Work under the Multiple Fellowship in Chemical Hygiene is being devoted mainly to the study of the safety of chemical residues remaining in food as a result of new developments in its production, processing and packaging, and the low level has been demonstrated of sensitizing ability of a new epoxy resin catalyst now being introduced. Under a fellowship in the effect of atmospheric moisture on air-borne bacteria a better understanding has been yielded of the water exchange between bacteria and their atmospheric environment and of the way in which certain chemicals alter this relation when bacteria are dispersed from suspensions of these substances. The Fellowship in Mine-Acid Control is now applying the accumulated information of nine years to a large complete watershed in a coal-mining area. The Multiple Fellowship in Glass Science has developed improved procedures for measuring the nuclear-spin relaxation-times of the silicon-29 atoms in glass, continued its study of irradiated glasses and commenced experimental studies on the surface of glass. The Multiple Fellowship in the Properties of Synthetic Rubber, now sponsored by the United States National Science Foundation, has scrutinized the determination of macromolecular sizes by methods of intrinsic viscosity and molecular weight and also the application of this technique to studies of branching in polymers. The degree of orientation caused by stretching the elastomer has also been investigated, and the response of solid rubber compounds to sinusoidal stresses. A filler has been developed that possesses a static buoyancy similar to that of buoyant glass fibres, in which the inherent strength, bulkiness and resiliency of a synthetic staple fibre of extremely small diameter are combined with the water repellency of a silicone polymer, and further tests are being made of the efficiency of this product. By combining xerography with electroluminescent powders, workers under the Multiple Fellowship on Carpenter Components have produced panels containing self-luminous information displays, which appear to be specially attractive for instrument panels.

About 60 per cent of the report is concerned with the research programmes of the Fellowships in Applied Science, and among the points of more general interest which may be mentioned are the fundamental studies on the ageing of selenium rectifier plates made under the Multiple Fellowship in Power Rectifiers and the construction of a furnace to grow single crystals of silicon in a vacuum or in an inert atmosphere with independent pulling and rotation of the seed crystal. A study of the interaction of organic ammonium salts with bentonite has led to a stabler clay complex which is organophilic and hydrophobic and is used in gelling oils to greases and in controlling the thixotropy of oil-base paints. Considerable success has been achieved in determ. ining the optimum water content for the proper extrusion of clay-water mixes by use of the 'Brabender plastograph', and an apparatus has been designed and constructed to measure the forcedeformation characteristics of green clay bars under transverse sheer. Progress has been made in the development of cover-coat enamels with improved alkali resistance for use on sheet steel. Several interesting organic derivatives of zirconium have been prepared, and the production of a metal zirconium plate by electrolysis in non-aqueous media investigated. A comprehensive information-proces- sing system, utilizing commercially available computers, communication systems and other devices, has been designed, and much basic research has been done on fundamental problems in the painting of steel structures, such as anchor-pattern profile, blastcleaning inhibitors, and cleaning agents. An investigation of natural sediment in streams was commenced, and progress has been made with paper chromatography as a method for differentiating phenolic compounds and in a study of odour thres. holds of pure phenolic compounds. Low-temperature crystallization techniques have been developed for the purification of organic compounds, and alloys of unusual characteristics have been developed by the electromelting of waste products from the recovery and utilization of anthracite.

Improved methods of synthesis have been devised. for certain of the porphyrins and their metallic complexes, and the structure of the vanadium complexes determined. Volatility studies have been made on some of these metallic complexes, and studies initiated on the thermal stability of various sulphur concentrates from crude petroleums. A chemical shock tube has been designed and constructed for a fundamental study of combustion reactions, and a new micro-catalytic chromatographic technique developed for studying catalytic reactions, which promises to accelerate the accumulation of data on new catalysts. An extensive study was made of the chemisorption of nitrogen, carbon dioxide and carbon monoxide on nickel catalysts, prepared in the presence of alkali, and studies were continued on the com. position and properties of fractions of high molecular weight from petroleum, while the use of calcium phosphates as a solid catalyst or catalyst carrier for various industrial reactions received detailed atten. tion. Phase-contrast photomicrographs have been taken of a wide variety of plain and modified starches before and after heat treatment, and several modifications have been made in the method of synthesizing the bacterial starter from which salt-rising bread is manufactured.

A new dextrin has been developed for use as a high-performance size for filament acetate, and methyl glucoside has been used in the preparation of long oil-modified alkyl resins, oil derivatives and modified amino-formaldehyde resins. Compounds have been discovered with excellent detergency at low concentration in perchloroethylene, and the synthesis and evaluation of soluble linear polymers were continued; a comprehensive study of the autoxidation of polyalkylene oxide derivatives has revealed that carbon-to-carbon bonds along the whole length of the polyalkylene oxide chains, as well as terminal hydroxy-alkyl groups, are vulnerable to oxidative attack. Considerable effort has been given to the synthesis of materials for use as, or in, high-temperature lubricating and hydraulic fluids, and more than fifty systems were examined for the stabilization of vinyl films to heat and light. The field of polyethylene/paraffin wax blends for coating paper-container stock was explored further, and methods were sought for determining the wettability of a metallic surface by a coating and the ability of a coating to flow on a metal surface. New techniques were developed for the preparation of functionally active siloxanes, and a special glow-proofed 'Neoprene' foam, which meets flame and smouldering-resistance requirements, was being developed. Besides the preparation of 6-diazo-5-oxo-L-norleucine, which is highly effective in inhibiting the growth of molignant 
tumours in mice and rats, studies of the mechanism by which the antibiotic, azaserine, inhibits the growth of malignant tumours showed that azaserine blocks at least one previously unknown enzyme from carrying out a key reaction involving I-glutamine and led to the identification of formylglycinamide ribotide as the reactant and formylglycinamidine ribotide as the product of the blocked reaction.

\section{SPACE PERCEPTION IN CHICKS}

TCKHARD H. HESS has carried out investigations 1 to ascertain whether a chick's visual perception of space depends upon learning or upon the maturation of an innate ability (Sci. Amer., July 1956).

Experiments were devised to prevent normal visual experience and which would not interfere with the normal physiological development of the eye. This was done by fitting the chicks' eyes with prismatic lenses which would displace the visual image to the right or to the left.

If the exact visual localization of objects in space is a totally learned ability, the chick's performance would be unaffected by the fact that it is wearing displacement prisms.

If, on the other hand, the chick is born with an innate ability to locate objects visually, the first pecks which such a chick directs toward objects seen through the displacement lenses would be about seven degrees to the right.

In experiments, twenty-eight Leghorn chicks were hatched in complete darkness and were immediately fitted with thin rubber hoods into which transparent plastic goggles had been inserted. The hoods were placed over their heads quickly in such a subdued light that the animals had essentially no normal light experience.

All the animals were returned to darkness for a period of about six hours so that they could become accustomed to the hoods. Then, when they were about one day old, all were tested for pecking accuracy. They were allowed to strike at small objects embedded in modelling clay. The targets were small brass nails, embedded so that they could not be dislodged by pecking.

There was a fundamental difference in the performance of control and experimental animals. In the control group the pecks were scattered about the target so that the target itself formed the centre of the distribution. For those chicks wearing lenses which displaced the visual field to the right, the pecks were similarly scattered; but they were centred about a point seven degrees to the right of the target. Similarly, the group the lenses of which displaced their visual images to the left showed a scattering of pecks to the left of the target.

Actual grain was now scattered and, when the chicks were between three and four days old, they were tested again. The results showed a great increase in accuracy on the part of the control chicks: now their pecks clustered quite closely about the target.

Among the animals wearing displacement prisms, improvement of a kind had also occurred. The pecks were clustered just as tightly as those of the controls, showing that increased accuracy had certainly been achieved.

It would appear that the chick's visual apparatus for locating objects in space is innate and not learned.
This conclusion is based on the fact that the chick wearing displacement prisms clustered its pecks about the spot where the object was seen. It did not peck at random until it struck the target.

Moreover, the chick the visual field of which was displaced appeared unable to learn through experience to correct its aim. Its only improvement was to increase the consistency of the distance by which it missed the target. Apparently, the innate picture which the chick has of the location of objects in its visual world cannot be modified through learning if what is required is that the chick learns to perform a response which is antagonistic to its instinctive one.

Further experiments showed that adult chickens and newly hatched chicks used binocular cues for localizing objects in space. This innate organization for the perception of depth requires neither learning nor continued use for its presence in the adult animal.

\section{HOME CARE AND NURSING}

$A^{1}$

$\mathrm{N}$ entire issue of The Practitioner is devoted to A home care and nursing (179, No. 1057; July 1956). In an introductory article Dr. G. E. Godber notes two features of the National Health Service in Britain which have tended to be overlooked. One is that the Service depends primarily upon comprehensive home care, with hospitals and specialist services only for diagnosis and treatment of illness outside the scope of the family doctor and his supporting team. The other is that "the greatest innovations of the Service have been the domiciliary consultation with a consultant and the general provision of home helps". In other words, as Dr. Metcalfe Brown points out in his article on the domiciliary services available to the doctor, "The preventive services and the hospital service are there to help the family doctor, not to displace or replace him".

From the inception of the National Health Service the hospital service has absorbed a large proportion of the not inconsiderable sum which the nation spends upon the Service. Thus, during 1953-54, according to the Guillebaud Report, the hospitals absorbed more than 61 per cent of the $£ 430$ million spent on the Service that year, compared with only a little more than 12 per cent spent on the general medical service. Even if the cost of the pharmaceutical service is included in that of the general medical service, the proportion is still only a little more than 21 per cent.

'There can be little doubt that if more attention were devoted to the development of the domiciliary services available to the general practitioner, the unnecessary load now carried by the hospitals would be radically reduced. Further, and equally important, the standard of general practice would be improved, to the greater benefit of the individual patient. The symposium provides an authoritative review of the services available to the general practitioner and of examples of how these services can be developed. The emphasis is upon the home care of children as these form such a vitally important part of the work of the family doctor; but all aspects of home care are integrated into the general picture. Three of the articles are written by medical officers of health. This is a sign of the increasing realization of the need for the closest possible co-operation between the medical officer of health, who is responsible for most of the domiciliary services, and the general practitioner, for whom these services are intended. 